\title{
The origin of Scorpius X-1
}

\author{
I. F. Mirabel ${ }^{1,2}$ and I. Rodrigues ${ }^{1}$ \\ 1 Service d'Astrophysique / CEA-Saclay, 91191 Gif-sur-Yvette, France \\ 2 Instituto de Astronoma y Fsica del Espacio/Conicet, Argentina
}

Received 17 October 2002/Accepted 1 December 2002

\begin{abstract}
We have used multi-wavelength observations of high precision to derive the space velocity and compute the orbit around the Galactic Centre of the prototype X-ray binary Scorpius X-1. An origin in the local spiral arm of the Milky Way is ruled out. The galactocentric kinematics of Scorpius X-1 is similar to that of the most ancient stars and globular clusters of the inner Galactic halo. Most probably, this low-mass X-ray binary was formed by a close encounter in a globular cluster. However, it cannot be ruled out that a natal supernova explosion launched Scorpius X-1 into an orbit like this from a birth place in the galactic bulge. In any case, the Galactocentric orbit indicates that Scorpius X-1 was formed more than 30 Myrs ago.
\end{abstract}

Key words. stars: individual: Scorpius X-1 - X-rays: binaries - astrometry

\section{Introduction}

Scorpius $\mathrm{X}-1$ was the first discovered and it is the brightest persistent extra-solar celestial X-ray source (Giacconi et al. 1962). It is the prototype low-mass X-ray binary composed of a compact object and a donor star that have masses of $1.4 M_{\odot}$ and $0.42 M_{\odot}$, respectively (Steeghs \& Casares 2002). The orbital period is of $18.9 \mathrm{hr}$ and the compact object is accreting matter from the Roche lobe-filling companion star. Although the mass of the compact object is consistent with that of a neutron star, no thermonuclear explosion (X-ray burst of type I) of the infalling matter on the putative hard surface of a compact object has been reported so far.

The kinematical properties of Sco X-1 can help us in understanding its origin. From the radial velocity, proper motion and distance of the system, the space velocity can be derived. Thereafter, using a mass model for the Galaxy, the galactic path can be tracked. This approach was applied for first time to derive the Galactocentric orbits of the halo black hole binary XTE J1118+480 (Mirabel et al. 2001) and the Galactic disk runaway black hole system GRO J1655-40 (Mirabel et al. 2002).

Scorpius $\mathrm{X}-1$ is a microquasar (for a review see Mirabel \& Rodríguez 1999), namely, a source of relativistic jets with a compact radio core associated to the accreting compact object (Fomalont et al. 2001). The most accurate proper motion of X-ray binaries can be obtained using Very Long Baseline Interferometry (VLBI) techniques at radio wavelengths. In the case of Scorpius X-1, a precise distance of $2.8 \pm 0.3 \mathrm{kpc}$ from the trigonometric parallax, and a proper motion of the radio

Send offprint requests to: I. F. Mirabel, e-mail: fmirabel@cea.fr core have been determined using VLBI (Bradshaw et al. 1999). Furthermore, an accurate value for the systemic radial velocity was recently reported by Steeghs \& Casares (2002) from spectroscopic observations at optical wavelengths. Because of the unprecedented precision in the distance, proper motion, and systemic radial velocity, Scorpius X-1 is the X-ray binary for which we have obtained the most accurate determination of the galactic path, among the seven X-ray binary systems studied so far (Mirabel \& Rodrigues 2002).

\section{Space velocity and galactocentric orbit}

The position, distance, heliocentric radial velocity, and proper motion are listed in Table 1. These data were used to compute the galactic orbit of Scorpius X-1 using the galactic gravitational potential model by Johnston et al. (1995). The velocity components $U, V$, and $W$, directed to the Galactic centre, rotation direction, and North Galactic pole were derived using Johnson \& Soderblom (1987)'s equations of transformation, and assuming the sun moves with components $\left(U_{\odot}, V_{\odot}, W_{\odot}\right)=$ $(9,12,7) \mathrm{km} \mathrm{s}^{-1}$ relative to the local standard of rest (LSR) (Mihalas \& Binney 1981). The values of $U, V$, and $W$ in Table 1 are rather different from the mean values that characterize the kinematics of stars that belong to the thin and thick disk of the Galaxy (Chiba \& Beers 2000).

The parameters and graphic representations of the galactocentric orbit are given at the end of Table 1 and in Fig. 1, respectively. Because the distance, proper motion and systemic radial velocity were determined with high precision, the errors in the orbital parameters of the galactocentric orbit of Scorpius X-1 are dominated by the uncertainties in the Galactic potential model. The errors for the orbital parameters quoted in Table 1 are the deviations derived from comparing the prescriptions by 
Table 1. Data and computed galactocentric orbital parameters for Scorpius X-1. References given in 3rd column are: $1=$ Bradshaw et al. (1999), 2 = Steeghs \& Casares (2002).

\begin{tabular}{|c|c|c|}
\hline$l\left[^{\circ}\right]$ & 0.72 & \\
\hline$b\left[^{\circ}\right]$ & +23.18 & \\
\hline$D[\mathrm{kpc}]$ & $2.8 \pm 0.3$ & 1 \\
\hline$V_{\text {helio }}\left[\mathrm{km} \mathrm{s}^{-1}\right]$ & $-113.8 \pm 0.6$ & 2 \\
\hline$\mu_{\alpha}\left[\operatorname{mas~yr}^{-1}\right]$ & $-6.88 \pm 0.07$ & 1 \\
\hline 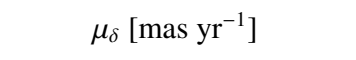 & $-12.02 \pm 0.16$ & 1 \\
\hline$U_{\mathrm{LSR}}\left[\mathrm{km} \mathrm{s}^{-1}\right]$ & $-93 \pm 2$ & \\
\hline$V_{\mathrm{LSR}}\left[\mathrm{km} \mathrm{s}^{-1}\right]$ & $-179 \pm 20$ & \\
\hline$W_{\mathrm{LSR}}\left[\mathrm{km} \mathrm{s}^{-1}\right]$ & $-74 \pm 4$ & \\
\hline Galactocentric eccentricity & $0.87 \pm 0.05$ & \\
\hline $\mathrm{Z}_{\max }[\mathrm{kpc}]$ & $4.2 \pm 0.2$ & \\
\hline$D_{\text {peri }}[\mathrm{kpc}]$ & $0.5 \pm 0.1$ & \\
\hline$D_{\text {apo }}[\mathrm{kpc}]$ & $7.4 \pm 0.4$ & \\
\hline
\end{tabular}

Flynn et al. (1996) and de Oliveira et al. (2002) with the one adopted by us (Johnston et al. 1995). The computed orbits that result from the use of the different current mass models for the Galaxy are very similar.

Scorpius X-1 does not participate in the Galactic rotation and its kinematics is unlike those of stars that belong to the thin and thick disk populations. Presently, Scorpius X-1 is moving towards the Galactic plane with a free fall velocity of $74 \mathrm{~km} \mathrm{~s}^{-1}$, and it is receding from the Galactic Centre at a spatial speed of $125 \mathrm{~km} \mathrm{~s}^{-1}$.

\section{Discussion}

We first consider the possibility that Scorpius X-1 may be associated with Gould's belt, which is a spur of the local spiral arm that has a size of a few hundred parsecs. Bradshaw et al. (1997) pointed out that Scorpius X-1 is beyond the Sco-Cen association. The new refined distance of $2.8 \mathrm{kpc}$ by Bradshaw et al. (1999) locates the X-ray source farther beyond the edge of the belt, which is expanding. Since Scorpius X-1 is approaching along the line of sight with a velocity of $113.8 \mathrm{~km} \mathrm{~s}^{-1}$ (see Table 1), the possibility that Scorpius X-1 has been formed and ejected from Gould's belt is ruled out.

Two possibilities remain: either Scorpius X-1 was kicked out into such an orbit by the explosion of the massive progenitor of the compact object, or it was formed in the halo itself. $30 \mathrm{Myr}$ ago the binary was at a perigalactic distance of $\sim 500 \mathrm{pc}$ with a velocity relative to the Galactic Centre of $\sim 480 \mathrm{~km} \mathrm{~s}^{-1}$, implying - after subtraction of Galactic rotation - a minimum kick velocity of $\sim 230 \mathrm{~km} \mathrm{~s}^{-1}$. This corresponds to a minimum linear momentum of $\sim 420 M_{\odot} \mathrm{km} \mathrm{s}^{-1}$. Although large, this minimum linear momentum does not exceeds the maximum linear momenta found among runaway solitary neutron stars and millisecond pulsars (Toscano et al. 1999). Besides, Brandt \& Podsiadlowski (1995) calculate that after a supernova explosion the resulting low mass $\mathrm{X}$-ray binary can remain bound provided it does not attain a velocity larger than $180 \pm 80 \mathrm{~km} \mathrm{~s}^{-1}$. The minimum kick velocity for Scorpius X-1 is at the limit of the maximum velocity for which the binary can remain bound. Unfortunately, from the theoretical point of view, the timescales after the explosion to achieve persistent Roche lobe overflow by the secondary and circularization of the binary orbit, are both rather uncertain. Therefore, although unlikely, one cannot rule out the possibility that Scorpius X-1 was launched from the Galactic bulge by the explosion of the progenitor of the compact object.

The galactocentric orbit of Scorpius X-1 is consistent with an origin in a globular cluster. The orbit is highly eccentric $(e=0.87)$, with a perigalactic distance of $0.5 \mathrm{kpc}$, and a maximum height above the plane of $4.2 \mathrm{kpc}$. It has comparable parameters to the orbits of globular clusters of the inner halo, such as NGC 6121 and NGC 6712 (Dauphole et al. 1996). However, we find that since the last perigalactic passage 30 Myrs ago, Scorpius X-1 did not intersect any known orbit of a globular cluster.

We point out that the majority of low-mass X-ray binaries of short period are found in globular clusters. Because of the high stellar densities and low velocity dispersions the cores of globular clusters are the best sites in the Galaxy for the formation by close encounters of this class of X-ray binaries. In this context, Scorpius X-1 most probably was formed in the core of a globular cluster in a close encounter of the compact object with a single star or with a binary (Verbunt 2002), being catapulted out of the core more than 30 Myrs ago.

\section{Conclusion}

Thanks to the unprecedented precision in the distance and proper motion from VLBI measurements by Bradshaw et al. (1999), and the accurate systemic radial velocity measured with optical spectroscopy by Steeghs \& Casares (2002), a precise orbital motion of Scorpius X-1 around the galactic centre was computed. The source reached a perigalactic distance of $500 \mathrm{pc}$ at a velocity of $480 \mathrm{~km} \mathrm{~s}^{-1}$. Presently, it is at a distance from the Sun of $2.8 \mathrm{kpc}$, falling to the Galactic plane with a vertical speed of $74 \mathrm{~km} \mathrm{~s}^{-1}$. A possible origin associated to the Gould's belt can now be ruled out. Scorpius X-1 has an eccentric orbit $(e=0.87)$ around the Galactic Centre, and the kinematics is similar to that of the most ancient stars and globular clusters of the inner halo. Most probably Scorpius X-1 has been formed in the core of a globular cluster, as the majority of the low mass $\mathrm{X}$-ray binaries of short orbital period. However, we cannot rule out that a natal supernova kick launched Scorpius X-1 into an orbit like this from a birth place in the galactic bulge. In the context of these two possibilities Scorpius X-1 must have been formed more than 30 Myrs ago. 

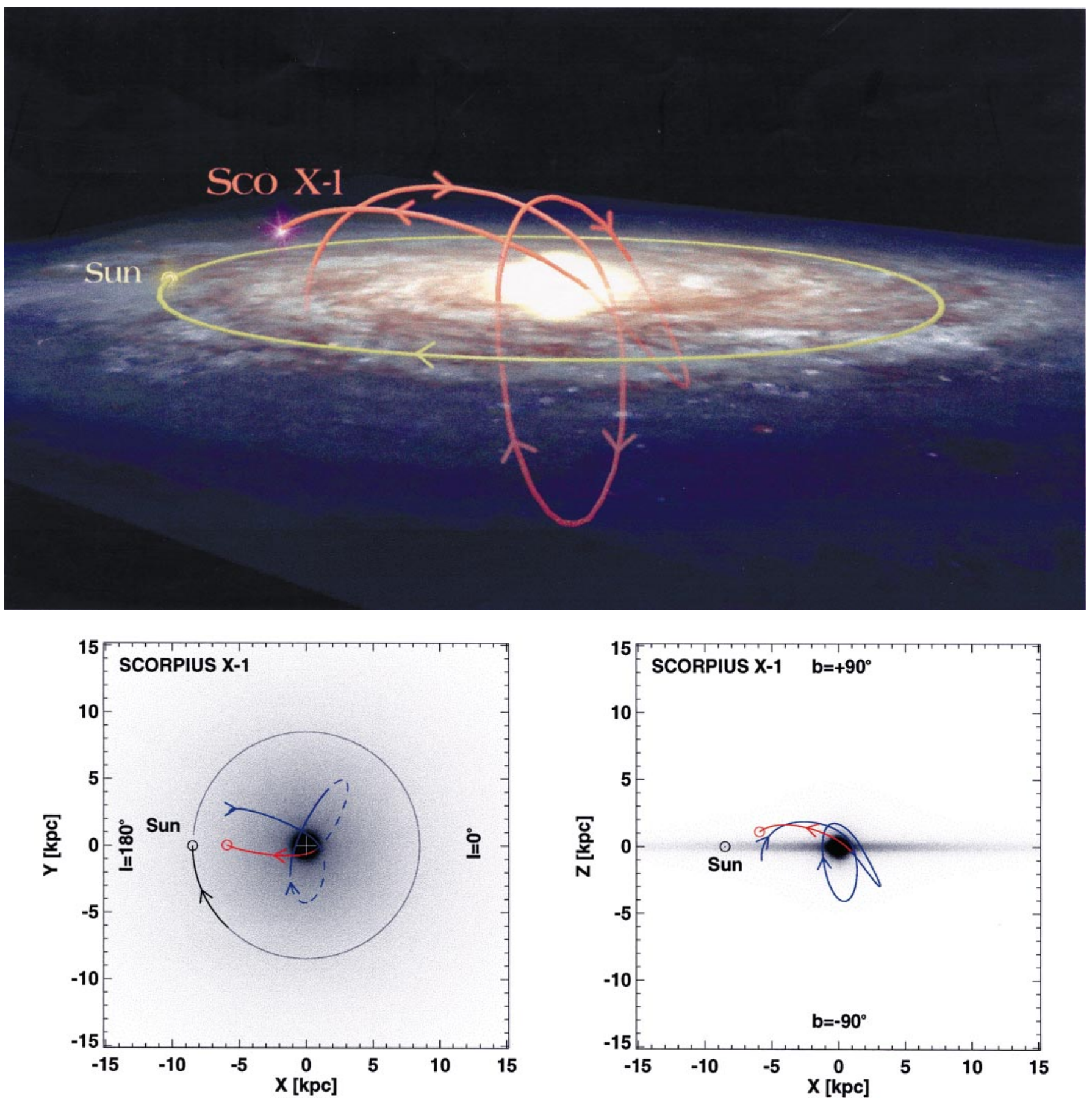

Fig. 1. Galactocentric orbit of Scorpius X-1. In the top panel is traced in red color the orbit of Scorpius X-1 around the Galactic Centre and in yellow the orbit of the Sun for a period of 230 Myrs. The lower two panels represent the view from above the Galactic plane (left panel) and the side view (right panel). In the left panel the continuous line represents the section of the orbit in the Northern Galactic Hemisphere; the section of the orbit in the Southern Hemisphere is shown by discontinuous trace. The orbit since the last perigalactic distance of $\sim 500 \mathrm{pc}$ that took place 30 Myrs ago is shown in red color. An animation of the orbital motion of Scorpius X-1 around the Galactic centre can be seen at: http://www.iafe.uba.ar/astronomia/mirabel/mirabel.html

Acknowledgements. We thank Jacques Paul and an anonymous referee for very useful comments on the original manuscript. I.F.M. is a member of the Consejo Nacional de Investigaciones Científicas y Técnicas of Argentina, and I.R. is a fellow of the Conselho Nacional de Desenvolvimento Cienífico e Tecnológico of Brazil. I.F.M. acknowledges support from PIP 0049/98 and Fundación Antorchas.

\section{References}

Bradshaw, C. F., Fomalont, E. B., \& Geldzahler, B. J. 1997, ApJ, 484, L55

Bradshaw, C. F., Fomalont, E. B., \& Geldzahler, B. J. 1999, ApJ, 512, L121

Brandt, N., \& Podsiadlowski, P. 1995, MNRAS, 274, 461

Chiba, M., \& Beers, T. C. 2000, AJ, 119, 2843 
Dauphole, B., Geffert, M., Colin, J., et al. 1996, A\&A, 313, 119

de Oliveira, M. R., Fausti, A., Bica, E., \& Dottori, H. 2002, A\&A, 390, 103

Flynn, C., Sommer-Larsen, J., \& Christensen, P. R. 1996, MNRAS, 281,1027

Fomalont, E. B., Geldzahler, B. J., \& Bradshaw, C. F. 2001, ApJ, 558, 283

Giacconi, R., Gursky, H., Paolini, F., \& Rossi, B. 1962, Phys. Rev. Lett., 9, 439

Johnson, D. R. H., \& Soderblom, D. R. 1987, AJ, 93, 864

Johnston, K. V., Spergel, D. N., \& Hernquist, L. 1995, ApJ, 451, 598

Mihalas, D., \& Binney, J. 1981, Galactic astronomy: Structure and kinematics - 2nd edition (San Francisco, CA, W. H. Freeman and Co.)
Mirabel, I. F., Dhawan, V., Mignani, R. P., Rodrigues, I., \& Guglielmetti, F. 2001, Nature, 413, 139

Mirabel, I. F., Mignani, R. P., Rodrigues, I., et al. 2002, A\&A, 395, 595

Mirabel, I. F., \& Rodrigues, I. 2002, in Proc. of the 4th Microquasar Workshop, 2002, ed. Ph. Durouchoux, Y. Fuchs \& J. Rodríguez, published by the Center for Space Physics: Kolkata

Mirabel, I. F., \& Rodríguez, L. F. 1999, ARA\&A, 37, 409

Steeghs, D., \& Casares, J. 2002, ApJ, 568, 273

Toscano, M., Sandhu, J. S., Bailes, M., et al. 1999, MNRAS, 307, 925

Verbunt, F. 2002 [astro-ph/0210057] 